\title{
MILLISECOND TIME VARIATIONS OF X-RAY BINARIES
}

\author{
J. H. SWANK \\ $N A S A / G S F C$ \\ Greenbelt,MD 20771, U.S.A.
}

\begin{abstract}
.
Millisecond time-scales are natural for some neutron star and black hole processes, although possibly difficult to observe. The Rossi X-Ray Timing Explorer (RXTE) has found that for the neutron stars in low-mass X-ray binaries (LMXB) there are flux oscillations at high frequencies, with large amplitudes. Z sources and bursters tend to exhibit oscillations in the range 300-1200 Hz. Persistent emission may exhibit one or both of two features. In bursts from different bursters, a nearly coherent pulsation is seen, which may be the rotation period of the neutron star. For some the frequency equals the difference between the two higher frequencies, suggesting a beat frequency model, but in others it is twice the difference. The sources span two orders of magnitude in accretion rate, yet the properties are similar. The similar maximum frequencies suggests that it corresponds to the Kepler orbit frequency at the minimum stable orbit or the neutron star surface, either of which would determine the neutron star masses, radii and equation of state. Theories of accretion onto black holes predict a quasi-periodic oscillation (QPO) related to the inner accretion disk. The two microquasar black hole candidates (BHCs) have exhibited candidates for this or related frequencies.
\end{abstract}

\section{Dynamical Time-scales of Compact Stars}

The Newtonian Keplerian frequency in an orbit at radius $\mathrm{R}$ about a compact mass $M$ is a measure within a factor of a few of the time scales of various other phenomena, such as free fall, or oscillations, that can occur closest to the compact object. For a $1.4 M_{\odot}$ neutron star with a $10 \mathrm{~km}$ radius, the frequency is $2500 \mathrm{~Hz}$, corresponding to a period of $0.4 \mathrm{~ms}$. For a $16 M_{\odot}$ black hole and $R=\frac{6 G M}{c^{2}}$, the frequency would be $127 \mathrm{~Hz}$ with a $7 \mathrm{~ms}$ 
period. The effective area of the Proportional Counter Array (PCA) (0.7 $\left.\mathrm{m}^{2}\right)$, the time resolution $(1 \mu \mathrm{s})$, the data modes, and the telemetry $(30-512$ kps are possible), were all required to study this regime.

It is clear from the results of RXTE that dynamical phenomena close to neutron stars and stellar black holes do generate X-ray signals which vary on the dynamical time-scales and that, furthermore, the signals are not greatly smeared by scattering before escaping. Thus we are able to see oscillations of various sorts which are in the expected frequency regimes.

The signals that have been found for neutron stars in the frequency range $50 \mathrm{~Hz}$ to $1300 \mathrm{~Hz}$, starting with the discovery in 4U 1728-34 [1], are very significant (in many cases more than 10 sigma). The highest quasiperiodic signal that has been reported is about $1220 \mathrm{~Hz}$. Many of the QPO features are narrow; they have $Q=\frac{\Delta \nu}{\nu}=100-1000$.

During the first year of the RXTE mission, two BHCs were very bright, GRO J1655-40 and GRS 1915+105. In both of these, a feature which qualifies as related to the dynamical time-scale of the inner accretion disk has been seen, $300 \mathrm{~Hz}$ and $67 \mathrm{~Hz}$, respectively [2]. While for the LMXB QPO the fractional root mean square (rms) power of the QPO were 1-20\%, for these $\mathrm{BHC}$ features the fractional rms amplitudes were less than $1 \%$.

\section{High Frequencies from Neutron Stars}

Three kinds of frequencies higher than previously known have been discovered in neutron star systems with RXTE observations. There are now at least 6 low-mass X-ray binary sources of Type I bursts in which almost coherent oscillations have been seen during the bursts. There are at least 14 low-mass X-ray binaries in which oscillations at frequencies ranging from $300 \mathrm{~Hz}$ to $1220 \mathrm{~Hz}$ have been seen in the persistent emission [3].

The pattern of oscillation behavior is similar in many bursts. When the data is folded on the period, the pulsed light curve is sinusoidal. A spot slightly hotter than the rest of the neutron star and rotating with it in and out of view would generate such an oscillation. The fractional root mean square amplitude of the oscillation can exceed $50 \%$ at the beginning of the burst and decreases as the burst flux rises, as would be consistent with the model of a burning front spreading on the neutron star. We think the periods (1.7-2.7 ms) probably indicate the pulsars in these LMXB[4].

The kilohertz oscillations in the persistent emission from LMXB present a simple spectrum in comparison to lower frequency power spectral densities (PSDs) of these sources. Just two frequencies dominate over other signals. Changes in the source luminosity usually cause frequency change and the two features move together with the difference approximately constant. An obvious candidate for the higher frequency is the Kepler frequency of matter 
TABLE 1. LMXB with Kilohertz Oscillations

\begin{tabular}{llllll}
\hline Source & $L_{x}\left(10^{38}\right)$ & $H z_{L}$ & $H z_{H}$ & $\Delta$ & $H z_{B}$ \\
\hline 4U 0614+09 & 0.05 & $326,400-800$ & $730-1145$ & 326 & \\
4U 1728-34 & 0.06 & $650-790$ & $500-1150$ & 363 & 363 \\
Aql X-1 & 0.08 & 750 & & & 549 \\
4U 1608-52 & 0.1 & $690,800-900$ & $900-1125$ & $60-230$ & \\
4U 1702-43 & 0.1 & 900 & 1180 & 280 & 330 \\
4U 1636-53 & 0.2 & $800-900$ & $1170-1216$ & 255 & 581 \\
4U 1735-44 & 0.4 & & 1149 & & \\
KS 1731-26 & $0.2 ?$ & 898 & $1159-1207$ & 260 & 524 \\
X 1744-29? & $0.2 ?$ & & & & 589 \\
4U 1820-30 & 0.6 & $546-796$ & 1066 & 270 & \\
GX 17+2 & 1 & $880-682$ & 988 & 306 & \\
Cyg X-2 & 1 & $490-530$ & $730-1020$ & 343 & \\
Sco X-1 & 1 & 634 & 926 & 292 & \\
GX 5-1 & 4 & $794-820$ & $1062-1133$ & 247 & \\
\hline
\end{tabular}

orbiting the neutron star at the inner edge of the accretion disk. Various mechanisms could define the inner edge of the disk. It could be (See [5] for extended discussion.) interaction with the magnetosphere, a sonic radius, or the marginally stable orbit, if the neutron star's surface is inside it. A lower frequency would be seen at an alias with the neutron star rotation. If there is a clump circulating at the inner radius of the disk, accretion from it along field lines to the neutron stars magnetic pole would be most enhanced at the nearest conjunction of the pole and the clump.

The sources with reported high frequency signals in the persistent emission are summarized in Table 1 (luminosity in $\operatorname{ergs~} \mathrm{s}^{-1}$, frequencies in the persistent emission $(\mathrm{L}$ and $\mathrm{H})$, the difference $(\Delta)$, and in the bursts $(\mathrm{B}))$. It can be seen that while for $4 \mathrm{U}$ 1728-34 the difference between the 2 high frequencies equals that of the bursts, for 4 other sources, the difference is approximately half the frequency seen during the bursts. However the burst period does not appear interpretable as the harmonic of the spin because of the high pulsed amplitude and the sinusoidal form.

The maximum frequencies appear to be all very close to each other. This suggested that the determination of the frequency must be dominated by neutron star characteristics and roughly independent of the accretion rate and the optical depths of the formation region. We argued that most likely the equation of state of nuclear matter does lead to neutron stars with radii inside the inner-most stable orbit for a neutron star, and the 
disk cannot approach closer to the neutron star than this[7]. The observed frequencies of about $1200 \mathrm{~Hz}$ would imply neutron star masses of $1.8-2 M_{\odot}$. The existence of an orbit which is marginally stable would be a verification of an important consequence of strong gravity[8].

\section{BHC oscillations}

The frequency of fast oscillations in the inner disk around a black hole depends on the black hole mass, its angular momentum, and the model. Instabilities of the disk which lead to circulating clumps would imply the masses of 7 and $33 M_{\odot}$ for GRO J1655-40 and GRS 1915+105, respectively, if they are slowly rotating. Epicyclic frequencies would match those observed for smaller slow black holes, but for the more massive black holes if they have near maximum rotation rate[6]. The mass and the angular momentum of the hole imply the inner radius of the optically thick accretion disk and the inner radius is more consistent with the mass of GRO J1655-4 deduced from optical measurements if the hole is rotating fast[9].

\section{References}

1. Strohmayer, T. E., Zhang, W., Swank, J. H., Smale, A. P., Titarchuk, L., \& Day, C. (1996) Millisecond X-Ray Variability from an Accreting Neutron Star System, Ap. J. Letters, 469, L9.

2. Remillard, R. E., Morgan, E. H., McClintock, J. E., Baiyln, C. D., Orosz, J. A., \& Greiner, J. (1997) Multifrequency Observations of the Galactic Microquasars GRS 1915+105 and GRO J1655-40, Proceedings of 18th Texas Symposium on Relativistic Astrophysics, Eds. A. Olinto, J. Frieman, and D. Schramm, World Scientific Press.

3. van der Klis, M. (1997) Kilohertz Quasi-Periodic Oscillations in Low-Mass X-Ray Binaries, Proc. NATO ASI "The Many Faces of Neutron Stars", in press.

4. Strohmayer, T. E., Zhang, W., \& Swank, J. H. (1997) $363 \mathrm{~Hz}$ Oscillations during the Rising Phase of Bursts from $4 \mathrm{U}$ 1728-34: Evidence for Rotational Modulation, $A p$. J. Letters, 487, L77.

5. Miller, M. C., Lamb, F. K., \&Psaltis, D. (1998) Sonic-Point Model for High-Frequency QPOs in Neutron Star Low-Mass X-Ray Binaries, Ap. J. Suppl., in press.

6. Nowak, M. A., Wagoner, R. V., Begelman, M. C., \& Lehr, D. E. (1997) The 67 $\mathrm{Hz}$ Feature in the Black Hole Candidate GRS $1915+105$ as a Possible Diskoseismic Mode, Ap. J. Letters, 477, L91.

7. Zhang, W., Strohmayer, T., \& Swank, J. H. (1997) Neutron Star Masses and Radii as inferred from Kilohertz Quasi-Periodic Oscillations, Ap. J. Letters, 482, L167.

8. Kaaret, P., Ford, E. C., \& Chen, K. Strong-Field General Relativity and Quasiperiodic Oscillations in X-Ray Binaries, Ap. J. Letters, 480, L27.

9. Zhang, S. N., Cui, W., \& Chen, W. (1997) Black Hole Spin in X-Ray Binaries: Observational Consequences, Ap. J. Letters, 482, L155. 